\title{
ESTIMATIVA DAS TEMPERATURAS MÉDIAS DIÁRIAS, DIURNAS E NOTURNAS A PARTIR DAS TEMPERATURAS HORÁRIAS
}

\author{
HAMILTON JUSTINO VIEIRA ${ }^{1}$, ÁLVARO JOSÉ BACK ${ }^{1,2}$, FÁBIO LOPES ${ }^{1}$, HEVERLY MORAIS ${ }^{1}$
}

${ }^{1}$ Empresa de Pesquisa Agropecuária e Extensão Rural de Santa Catarina, Centro de Informações de Recursos

Ambientais e de Hidrometeorologia (EPAGRI/CIRAM), Florianópolis, SC, Brasil

${ }^{2}$ Universidade do Extremo Sul Catarinense (UNESC), Criciúma, SC, Brasil

hamiltonjv@gmail.com, ajb@epagri.sc.gov.br, fzlopes1@gmail.com,heverly@iapar.br

Recebido Abril de 2010 - Aceito Fevereiro 2012

\begin{abstract}
RESUMO
É amplamente conhecido atualmente, que as temperaturas diurnas e ou noturnas são fatores decisivos na exploração de determinadas culturas. Contudo, a determinação destas foi sempre dificultada pelo modo que eram realizadas as leituras meteorológicas. Este trabalho teve como objetivo ajustar equações para estimativa das temperaturas médias noturnas (TN), diurnas (TD) e a média diária (Tm) em função das temperaturas horárias instantâneas. Foram utilizados dados horários da temperatura instantânea da estação automática de São Joaquim, SC (Lat. 2816'54" S, Long. 4956'07” W, Alt. 1408m) relativo ao ano de 2006. A partir dos dados de temperaturas instantâneas horárias foram calculados os valores de TD, TN e Tm. O período diurno foi definido como sendo das 7 às 19 horas. Foram realizadas análises de regressão linear tendo como variável independente os valores de temperatura instantânea, e variável dependente os valores de TD, TN e Tm, sendo o melhor estimador definido pelo maior valor do coeficiente de determinação. Foram selecionados os valores de temperatura instantâneas das $11 \mathrm{~h}, 24 \mathrm{~h}$ e $20 \mathrm{~h}$ como estimadores das TD, TN e Tm, respectivamente. Também foram ajustadas equações gerais para estimativa dos valores de TD, TN e Tm em função das temperaturas instantâneas de qualquer horário.
\end{abstract}

Palavras-chave: modelagem climática, regressão linear, temperatura do ar.

\section{ABSTRACT: AVERAGE DAILY, DAYTIMEAND NIGHTTIME TEMPERATURES ESTIMATION FROM HOURLY TEMPERATURES}

It is widely known that daytime and nighttime temperatures are decisive factors for the exploration of certain crops; however their determination has always been difficult due to the way by which the meteorological readings were carried out. This study aims to adjust equations to estimate average nighttime temperatures (NT), daytime temperatures (DT), and daily average (AT) according to the instantaneous hourly temperatures. We used hourly data of instantaneous temperature during 2006 from the automatic station of São Joaquim, SC (Lat. $28^{\circ} 16^{\prime} 54^{\prime \prime}$ 'S, Long. 49 $56^{\prime} 07^{\prime \prime} \mathrm{W}$, Alt 1408m). From the hourly instantaneous temperature data, values of DT, NT and AT were calculated. The daytime period was defined as being from 7:00 am to 7:00 pm. Linear regression analyses were done with the values of instantaneous temperature as independent variable and values of DT, NT and AT as dependent variables, and the best estimator was defined by the highest coefficient of determination. We selected the values of instantaneous temperature of 11:00 am, 12:00 am, and 8:00 pm as predictors of DT, NT and AT, respectively. General equations for DT, NT and AT estimation were also adjusted to the instantaneous temperatures at any time.

Keywords: climatic modeling, linear regression, temperature of air. 


\section{INTRODUÇÃO}

A grande maioria das culturas agrícolas possui o crescimento e o desenvolvimento afetados com maior ou menor intensidade pela variabilidade diária e sazonal da temperatura. O crescimento e desenvolvimento de várias espécies de vegetais estão associados às temperaturas, existindo temperaturas limites inferior e superior de crescimento, chamadas de temperaturas basais (Camargo et al., 1987, Pedro Junior et al., 1994). Vários índices biometeorológicos foram desenvolvidos para relacionar o comportamento fenológico de espécies vegetais com a temperatura do ar (Sentelhas, 1998; Tonietto e Carboneau, 2004). Dentre estes índices destacam-se o Índice hélio térmico de Huglin (IH) (Conceição e Tonietto, 2005), Índice de Frio noturno (IF) (Tonietto, 1999), e o Graus-Dia-Desenvolvimento (GDD) (Murakami et al., 2002). O conceito de GDD é um índice bastante usado em videira e possui a vantagem da fácil determinação pela aquisição de dados de temperatura (Roberto et al., 2005). Embora seja amplamente conhecido que as temperaturas diurnas e/ou noturnas são fatores decisivos na exploração de determinadas culturas, a determinação destas foi sempre dificultada pelo modo pelo qual eram feitas as leituras meteorológicas. Estes dados, pela sua simplicidade ou pela generalidade de coleta, apresentam dificuldades de utilização devido ao grande esforço para digitalização e digitação. $\mathrm{Na}$ década de 90 surgiram as primeiras estações meteorológicas automáticas. Com o funcionamento concomitante destas estações meteorológicas, foi possível implementar um detalhamento dos dados históricos e presentes, facilitando o uso daqueles provenientes das estações convencionais objetivando verificar a possibilidade de recuperar dados históricos.

Brunini et al. (1997) estudaram a viabilidade técnica e performance de uma estação meteorológica automática Campbell. Estes autores concluíram que a concepção adotada nas estações meteorológicas automáticas e os sensores, em uso, tem apresentado acurácia e precisão com custo não muito elevado, permitindo o uso nas diversas instituições do Brasil. Fietz et al. (1999) realizaram uma análise comparativa, em caráter preliminar, de dados obtidos por estações meteorológicas convencionais e automáticas, em Dourados-MS e concluíram que a temperatura máxima, mínima e média dos dois tipos de estação tem uma boa concordância. No entanto, Fisch e Santos (1997) apresentaram um estudo preliminar com os valores obtidos por estações meteorológicas automáticas e concluíram que estes diferem daqueles apresentados por estações convencionais e que o cálculo da temperatura do ar com dados da estação convencional superestima o valor verdadeiro em aproximadamente $1^{\circ} \mathrm{C}$. Sentelhas et al. (1997) fizeram um estudo comparativo semelhante e concluíram que coeficientes de correlação da temperatura máxima, mínima e a média foram significativos a $1 \%$, e que a substituição da estação convencional pela automática não acarretará em mudanças significativas na série de dados. Araújo e Rodrigues (1998) concluíram que, devido à estação convencional de Brasília-DF estar bem calibrada, as diferenças apresentadas nos parâmetros analisados entre ela e a automática foram pequenas. Souza et al. (2002) concluíram que para a região de Maringá-PR com uma série de 34 meses de observação, as temperaturas máximas, mínimas e médias têm uma boa concordância entre ambas as estações.

Ferreira et al. (2002), com estudo na Fazenda Água Limpa da Universidade de Brasília, concluíram que as temperaturas máximas da estação automática apresentam tendência a serem inferiores aos da estação convencional. Já a temperatura mínima apresentou uma boa relação entre as duas estações nestes dois meses analisados. Cunha e Martins (2004), em Botucatu-SP, concluíram que a concordância entre as variáveis de ambas as estações são boas com exceção da velocidade do vento. Sentelhas et al. (1997) não recomendam a troca imediata da estação meteorológica convencional pela automática devido aos elevados custos. Recomendam também, que seja feita uma análise comparativa das duas estações por um período representativo. Scarpare et al. (2006), comparando a uniformidade dos dados registrados na estação convencional e automática localizadas no campus da Escola Superior de Agricultura Luiz de Queiroz (ESALQ), da unidade da Universidade São Paulo (USP) em Piracicaba-SP, concluíram que os elementos meteorológicos com maior uniformidade entre as estações foram a temperatura média do ar e a precipitação. No decorrer das últimas décadas houve a intensificação da agricultura, necessidade de maior eficiência de produção, maior competitividade, diminuição e ou extinção das novas fronteiras agrícolas e as mudanças climáticas globais. Diante desta realidade se faz necessário o refinamento dos estudos agrometeorológicos para dar respostas à demanda pelo aprimoramento e melhoria do manejo agropecuário. $\mathrm{O}$ acesso às temperaturas diurnas e noturnas é amplamente utilizado na viticultura, cultivo da macieira, do alho, da cebola e da batata, entre outras. Este trabalho teve como objetivo comparar as temperaturas do ar obtidas por uma Estação Meteorológica Convencional com uma Estação Meteorológica Automática e avaliar os métodos de estimativa das temperaturas médias diárias e das temperaturas médias diurna e noturna.

\section{MATERIAL E MÉTODOS}

Foram utilizados dados horários das temperaturas horárias do ano de 2006, da estação automática de São Joaquim-SC, com Lat. $28^{\circ} 16^{\prime} 54^{\prime \prime}$ S, Long. 49 56'07" W e Alt. $1408 \mathrm{~m}$, pertencente à Empresa de Pesquisa Agropecuária e de Extensão Rural de Santa Catarina (Epagri). O clima da região, segundo classificação de 
Tabela 1 - Médias das temperaturas máximas e mínimas do ar de São Joaquim-SC

\begin{tabular}{lcccccccccccc}
\hline Temperatura & Jan. & Fev. & Mar. & Abr. & Mai. & Jun. & Jul. & Ago. & Set. & Out. & Nov. & Dez. \\
\hline Máxima $\left({ }^{\circ} \mathrm{C}\right)$ & 22,8 & 22,3 & 21,6 & 19 & 15,6 & 14,7 & 14,3 & 16,3 & 16,4 & 18,8 & 20,4 & 22,0 \\
Mínima $\left({ }^{\circ} \mathrm{C}\right)$ & 13,4 & 13,5 & 12,7 & 10,5 & 7,4 & 6,6 & 5,7 & 7,0 & 7,1 & 9,3 & 10,5 & 12,2
\end{tabular}

Köppen, é mesotérmico úmido com inverso temperado $(\mathrm{Cfb})$. A Tabela 1 mostra as médias normais para a estação de São Joaquim-SC. Os dados horários de temperatura foram coletados em uma estação meteorológica automática, equipada com Datalogger CR510 (Campbell Sci. Inc., Logan, UT, USA), com sensor de temperatura com escala de -20 a $+50^{\circ} \mathrm{C}$ e umidade relativa do ar (UR) de 0 à $100 \%$, com erro de $0,2 \%$. Com os dados das temperaturas horárias foram calculadas as temperaturas médias do período diurno (TD) e do período noturno (TN), assim como as temperaturas médias do dia (Tm).

De posse destes valores foram realizadas análises de regressão entre e a TD, TN, Tm com as temperaturas horárias diárias. O modelo adotado foi de regressão linear, tendo como expressão:

$$
Y=\alpha+\beta X
$$

em que $\mathrm{Y}=$ variável dependente (TD, TN e Tm);

$\alpha=$ intercepto da equação;

$\beta=$ coeficiente angular;

$\mathrm{X}=$ variável independente (temperatura horária instantânea).

\section{RESULTADOS E DISCUSSÃO}

\subsection{Temperaturas diurnas}

A Tabela 2 mostra os coeficientes de determinação entre as temperaturas instantâneas horárias e as temperaturas médias do período diurno (TD), para São Joaquim-SC. Nota-se que os coeficientes de determinação ficaram acima de 0,80 onde o $\left(\mathrm{R}^{2}\right)$ mais elevado, de 0,96 , foi com a temperatura das 11 horas (Figura 1).

Tomando-se os valores da equação para o horário das 11 horas, que apresentou o maior coeficiente de determinação, pode-se estimar as temperaturas médias diurnas (TD) com a equação de regressão conforme Tabela $2 \mathrm{e}$ Figura 1. Em função dos altos valores do coeficiente de determinação para todos os horários, as temperaturas instantâneas das 9 às 15 horas e as temperaturas máximas podem também ser utilizadas nesta estimativa, já que são variáveis disponíveis na maioria das estações meteorológicas convencionais. Para a estimativa das médias das temperaturas durante o período diurno com estes dois horários, 9 e 15 horas, o coeficiente de determinação $\left(\mathrm{R}^{2}\right)$ foi de 0,93 e 0,89 , respectivamente. Considerando a média das

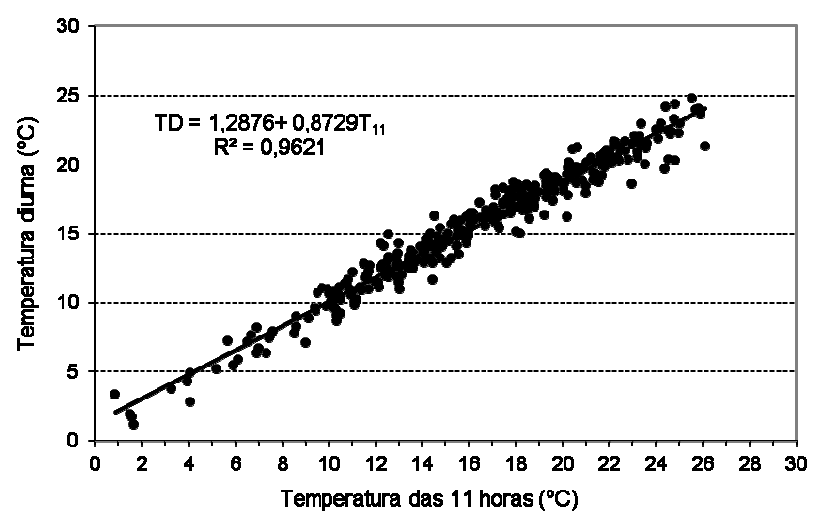

Figura 1 - Relação entre a temperatura instantânea das 11 horas (T11) e a média diária das temperaturas diurnas (TD) para São Joaquim-SC.

temperaturas das 9 e 15 horas obteve-se um ajuste ainda melhor $\left(\mathrm{R}^{2}=0,99\right)$.

Conforme se visualiza na Figura 2 a variação do coeficiente $\alpha$ da equação da reta de estimativa das temperaturas médias diurnas para os diversos horários, entre 7 e 19 horas apresentam uma variação exponencial de terceira ordem, com um coeficiente de determinação $\left(\mathrm{R}^{2}\right)$ de 0,97 , em que h é a hora do dia em que é realizada a leitura da temperatura instantânea $[7 \leq \mathrm{h} \leq 19]$.

Realizando a análise de regressão do coeficiente $\beta$ com a hora da leitura observa-se que o modelo não foi significativo ( $\mathrm{p}$ $=0,740$ ); sendo assim pode-se utilizar o valor médio de 0,8657 para todos os horários (Figura 3). Observa-se na Tabela 2 que o valor médio somente não está dentro do intervalo de confiança para os horários da $13 \mathrm{~h}$ e $17 \mathrm{~h}$. Com esses valores pode-se apresentar a equação geral para estimativa da temperatura diurna como:

$$
\begin{aligned}
& T D=0,0014 h^{3}+0,0785 h^{2}-2,9722 h+22,5206+0,8657 T h \\
& T D=0,0014 h^{3}+0,0785 h^{2}-2,9722 h+22,5206+0,8657 T h
\end{aligned}
$$

em que: $\mathrm{TD}=$ temperatura média diurna; $\mathrm{h}=$ hora da leitura $(7$ a 19); $\mathrm{Th}=$ temperatura da hora $\mathrm{h}$.

\subsection{Temperaturas noturnas}

Na Tabela 3 consta o resumo da análise de regressão entre as temperaturas médias noturnas (TN) e as temperaturas 
Tabela 2 - Coeficientes de determinação $\left(\mathrm{R}^{2}\right)$ entre a temperatura diurna (TD) e diferentes temperaturas instantâneas de horários diferentes, assim como os coeficientes " $\alpha$ " e " $\beta$ " da estação automática de São Joaquim-SC, com respectivos intervalos de confiança de $95 \%$.

\begin{tabular}{|c|c|c|c|c|c|c|c|}
\hline \multirow[b]{2}{*}{ Hora } & \multirow[b]{2}{*}{$\mathrm{R}^{2}$} & \multirow[b]{2}{*}{$\alpha$} & \multicolumn{2}{|c|}{ Intervalo de confiança (95\%) } & \multirow[b]{2}{*}{$\beta$} & \multicolumn{2}{|c|}{ Intervalo de Confiança } \\
\hline & & & $\alpha 1$ & $\alpha 2$ & & $\beta 1$ & $\beta 2$ \\
\hline 7 & 0,812 & 5,7473 & 5,2120 & 6,2826 & 0,8439 & 0,8018 & 0,8859 \\
\hline 8 & 0,875 & 4,6143 & 4,1503 & 5,0784 & 0,8598 & 0,8262 & 0,8935 \\
\hline 9 & 0,928 & 3,3668 & 2,9912 & 3,7424 & 0,8667 & 0,8418 & 0,8917 \\
\hline 10 & 0,946 & 2,2378 & 1,8906 & 2,5850 & 0,8662 & 0,8449 & 0,8875 \\
\hline 11 & 0,962 & 1,2876 & 0,9785 & 1,5967 & 0,8729 & 0,8550 & 0,8909 \\
\hline 12 & 0,959 & 0,4334 & 0,0970 & 0,7698 & 0,8802 & 0,8615 & 0,8989 \\
\hline 13 & 0,942 & $-0,2476$ & $-0,6699$ & 0,1747 & 0,8924 & 0,8696 & 0,9152 \\
\hline 14 & 0,913 & $-0,1847$ & $-0,7100$ & 0,3406 & 0,8730 & 0,8451 & 0,9009 \\
\hline 15 & 0,892 & 0,1456 & $-0,4318$ & 0,7231 & 0,8596 & 0,8288 & 0,8904 \\
\hline 16 & 0,876 & 0,6025 & $-0,0083$ & 1,2133 & 0,8567 & 0,8233 & 0,8901 \\
\hline 17 & 0,855 & 1,9259 & 1,3124 & 2,5395 & 0,8293 & 0,7940 & 0,8647 \\
\hline 18 & 0,882 & 3,0457 & 2,5424 & 3,5489 & 0,8450 & 0,8130 & 0,8769 \\
\hline 19 & 0,902 & 3,2495 & 2,8025 & 3,6965 & 0,9081 & 0,8773 & 0,9390 \\
\hline média & 0,903 & 2,0172 & 1,5505 & 2,4840 & 0,8657 & 0,8371 & 0,8943 \\
\hline \multicolumn{8}{|l|}{ média } \\
\hline 9 e 15 & 0,985 & 0,6108 & 0,4091 & 0,8125 & 0,9337 & 0,9217 & 0,9457 \\
\hline
\end{tabular}

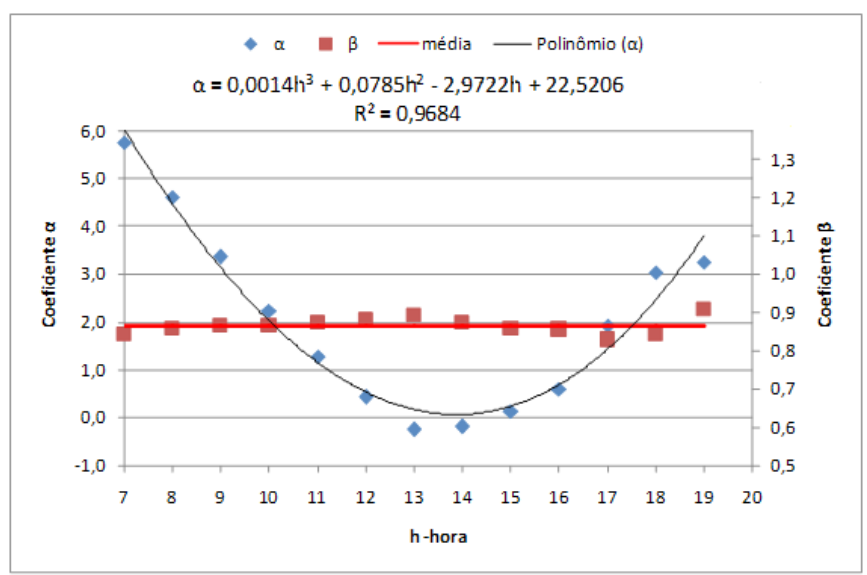

Figura 2 - Variação dos coeficientes " $\alpha$ " e " $\beta$ " para a estimativa das temperaturas diurnas com as temperaturas horárias instantâneas entre as 7 e 19 horas para a estação automática de São Joaquim-SC.

horárias instantâneas para São Joaquim-SC, entre 20 horas do dia considerado e 6 horas do dia seguinte. $\mathrm{O}$ maior coeficiente de determinação $\left(\mathrm{R}^{2}=0,983\right)$ foi obtido com a temperatura das 24 horas, sendo que a equação para a estimativa da temperatura noturna pode ser observada na Figura 3.

Considerando ainda os altos valores de $\mathrm{R}^{2}$ obtidos, pode-se concluir que os valores da temperatura instantânea das $21 \mathrm{~h}$, ou a temperatura mínima normalmente medidas nas estações convencionais, podem ser utilizados para a estimativa da temperatura noturna. Na Figura 4 estão representados os valores dos coeficientes $\alpha$ e $\beta$ da equação de regressão linear para estimativa das temperaturas médias noturnas (TN) em função das temperaturas horárias. Aplicando a análise de regressão entre o coeficiente $\alpha$ e o horário, obteve-se duas equações quadráticas, válidas para $1 \leq \mathrm{h} \leq 6$ horas $\left(\mathrm{R}^{2}=0,995\right)$ e para $20 \leq \mathrm{h} \leq 24$ horas $\left(\mathrm{R}^{2}=0,982\right)$, dadas respectivamente por:

$$
\alpha=-0,025 h^{2}+0,301 h+0,610 \text {, válida para } 1 \leq \mathrm{h}
$$
$\leq 6$ horas

$$
\alpha=-0,024 h^{2}+1,350 h-17,74, \text { válida para } 20 \leq \mathrm{h}
$$
$\leq 24$ horas

Já para o coeficiente $\beta$ a regressão não se mostrou significativa $(\mathrm{p}=0,1346)$ de forma que pode-se utilizar o valor médio de 0,9588 . Dessa forma, as equações para estimativa das temperaturas noturnas a partir das temperaturas instantâneas a qualquer horário, válida para $1 \leq \mathrm{h} \leq 6$ horas e válida para 20 $\leq \mathrm{h} \leq 24$ horas, são dadas respectivamente por:

$$
\begin{aligned}
& \mathrm{TN}=\left(-0,025 \mathrm{~h}^{2}+0,301 \mathrm{~h}-0,610\right)+0,9588 \mathrm{Th} \\
& \mathrm{TN}=\left(-0,024 \mathrm{~h}^{2}+1,350 \mathrm{~h}-17,740\right)+0,9588 \mathrm{Th}
\end{aligned}
$$

\subsection{Temperaturas médias diárias}

Na Tabela 4 consta o resumo das análises de regressão da temperatura média diária (Tm) em função das temperaturas 
Tabela 3 - Coeficientes de determinação (R2) entre a temperatura diurna (TN) e diferentes temperaturas instantâneas de horários diferentes (Th), assim como os coeficientes $\alpha$ e $\beta$ da estação automática de São Joaquim-SC, com respectivos intervalos de confiança de $95 \%$.

\begin{tabular}{cccccccc}
\hline & & \multicolumn{3}{c}{ Intervalo de Confiança } & & \multicolumn{2}{c}{ Intervalo de Confiança } \\
Hora & $\mathrm{R}^{2}$ & $\alpha$ & $\alpha 1$ & $\alpha 2$ & $\beta$ & $\beta 1$ & $\beta 2$ \\
\hline 20 & 0,956 & $-0,3038$ & $-0,5955$ & $-0,0121$ & 0,9596 & 0,9383 & 0,9809 \\
21 & 0,971 & $-0,0606$ & $-0,2920$ & 0,1707 & 0,9678 & 0,9505 & 0,9851 \\
22 & 0,980 & 0,3458 & 0,1580 & 0,5335 & 0,9603 & 0,9460 & 0,9747 \\
23 & 0,982 & 0,6839 & 0,5126 & 0,8551 & 0,9528 & 0,9395 & 0,9661 \\
24 & $\mathbf{0 , 9 8 3}$ & 0,7931 & 0,6251 & 0,9612 & 0,9576 & 0,9444 & 0,9709 \\
1 & 0,979 & 0,8903 & 0,7068 & 1,0737 & 0,9635 & 0,9489 & 0,9782 \\
2 & 0,972 & 1,1065 & 0,8979 & 1,3151 & 0,9605 & 0,9436 & 0,9774 \\
3 & 0,965 & 1,2702 & 1,0381 & 1,5024 & 0,9592 & 0,9401 & 0,9782 \\
4 & 0,951 & 1,4309 & 1,1592 & 1,7026 & 0,9566 & 0,9341 & 0,9791 \\
5 & 0,944 & 1,4572 & 1,1655 & 1,7489 & 0,9599 & 0,9356 & 0,9842 \\
6 & 0,935 & 1,4945 & 1,1816 & 1,8073 & 0,9494 & 0,9235 & 0,9754 \\
\hline média & 0,9653 & 0,8280 & 0,5961 & 1,0598 & 0,9588 & 0,9404 & 0,9773 \\
\hline
\end{tabular}

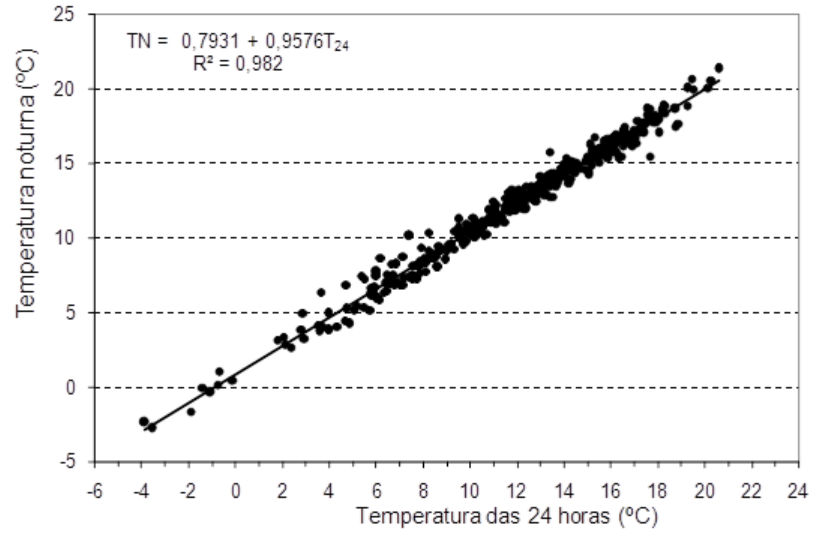

Figura 3 - Relação entre a temperatura noturna e a temperatura das 24 horas para São Joaquim-SC.

horárias (Th) para São Joaquim, SC. O melhor ajuste $\left(\mathrm{R}^{2}=\right.$ $0,949)$ foi obtido com a temperatura das 20 horas $\left(\mathrm{T}_{20}\right)$ (Figura $5)$.

Para o coeficiente $\beta$ a análise de regressão mostrou não haver diferença significativa em relação ao horário $(\mathrm{p}=0,1771)$ e, portanto, pode-se utilizar o valor médio 0,8742. Dessa forma, a equação para estimativa da temperatura média diária pode ser expressa por:

$\operatorname{Tm}=\left(-0,0004 h^{4}+0,0238 h^{3}-0,3998 h^{2}+1,9691 h+\right.$ $1,401)+0,8742 \mathrm{Th}$.

Os resultados acima apresentados concordam com Brunini et al. (1997), Fisch e Santos (1997), Sentelhas et al. (1997), Araújo e Rodrigues (1998), Fietz et al.(1999), Ferreira et al. (2002), Souza et al. (2002) e Cunha e Martins (2004),

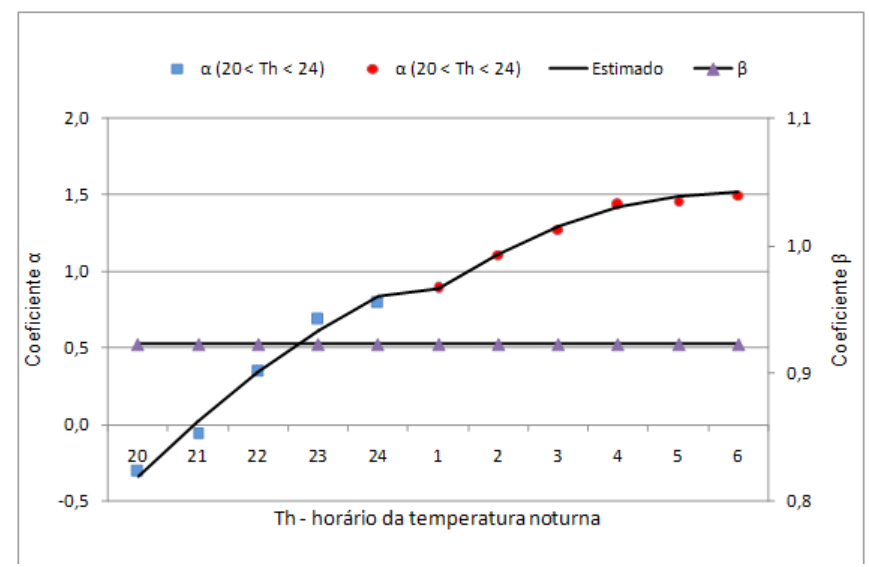

Figura 4 - Evolução temporal dos coeficientes $\alpha$ e $\beta$, da regressão linear da temperatura noturna em função da temperatura horária instantânea, das 20 horas às 6 da manhã.

que também obtiveram uma boa correlação entre as estações meteorológicas convencionais e meteorológicas. Por outro lado, este trabalho concorda com o de Brunini et al. (1997) e de Cunha e Martins (2004), que antes de proporem que a temperatura diurna seja obtida pela temperatura das $11 \mathrm{~h}$ da estação automática, a noturna pela das $24 \mathrm{~h}$ e a média pela das $20 \mathrm{~h}$, recomendam se utilizar um período maior de dados para verificar se as mesmas se concretizam e também verificar a acurácia dos sensores das estações meteorológicas automáticas.

$\mathrm{Na}$ Figura 6 estão representados os valores dos coeficientes $\alpha$ e $\beta$ obtidos com as diversas regressões em função do horário da temperatura instantânea. Para o valor de $\alpha$ foi ajustada uma equação polinomial de quarta ordem. 
Tabela 4 - Coeficientes de determinação $\left(\mathrm{R}^{2}\right)$ entre a temperatura média diária (Tmed) e diferentes temperaturas instantâneas de horários diferentes, assim como, os coeficientes " $\alpha$ " e " $\beta$ ” da estação automática de São Joaquim-SC com respectivos intervalos de confiança de $95 \%$.

\begin{tabular}{|c|c|c|c|c|c|c|c|}
\hline \multirow[b]{2}{*}{ Hora } & \multirow[b]{2}{*}{$\mathrm{R}^{2}$} & \multirow[b]{2}{*}{$\alpha$} & \multicolumn{2}{|c|}{ Intervalo de Confiança } & \multirow[b]{2}{*}{$\beta$} & \multicolumn{2}{|c|}{ Intervalo de Confiança } \\
\hline & & & $\alpha 1$ & $\alpha 2$ & & $\beta 1$ & $\beta 2$ \\
\hline 1 & 0,798 & 3,537 & 2,9668 & 4,1074 & 0,873 & 0,8273 & 0,9184 \\
\hline 2 & 0,802 & 3,670 & 3,1133 & 4,2262 & 0,876 & 0,8306 & 0,9207 \\
\hline 3 & 0,808 & 3,744 & 3,2016 & 4,2868 & 0,881 & 0,8366 & 0,9256 \\
\hline 4 & 0,803 & 3,854 & 3,3076 & 4,4011 & 0,882 & 0,8368 & 0,9274 \\
\hline 5 & 0,814 & 3,773 & 3,2413 & 4,3050 & 0,895 & 0,8502 & 0,9389 \\
\hline 6 & 0,834 & 3,640 & 3,1374 & 4,1417 & 0,900 & 0,8582 & 0,9413 \\
\hline 7 & 0,873 & 3,512 & 3,0759 & 3,9477 & 0,866 & 0,8316 & 0,9001 \\
\hline 8 & 0,905 & 2,567 & 2,1661 & 2,9678 & 0,865 & 0,8362 & 0,8944 \\
\hline 9 & 0,931 & 1,462 & 1,0972 & 1,8268 & 0,861 & 0,8372 & 0,8856 \\
\hline 10 & 0,921 & 0,545 & 0,1252 & 0,9647 & 0,848 & 0,8220 & 0,8734 \\
\hline 11 & 0,912 & $-0,204$ & $-0,6719$ & 0,2630 & 0,843 & 0,8162 & 0,8704 \\
\hline 12 & 0,887 & $-0,842$ & $-1,4020$ & $-0,2824$ & 0,840 & 0,8085 & 0,8706 \\
\hline 13 & 0,864 & $-1,431$ & $-2,0754$ & $-0,7861$ & 0,848 & 0,8129 & 0,8826 \\
\hline 14 & 0,825 & $-1,264$ & $-2,0049$ & $-0,5229$ & 0,823 & 0,7839 & 0,8626 \\
\hline 15 & 0,797 & $-0,866$ & $-1,6540$ & $-0,0778$ & 0,806 & 0,7640 & 0,8481 \\
\hline 16 & 0,790 & $-0,502$ & $-1,2913$ & 0,2865 & 0,807 & 0,7640 & 0,8502 \\
\hline 17 & 0,798 & 0,513 & $-0,2057$ & 1,2320 & 0,795 & 0,7540 & 0,8368 \\
\hline 18 & 0,877 & 1,198 & 0,6863 & 1,7100 & 0,836 & 0,8040 & 0,8689 \\
\hline 19 & 0,935 & 1,144 & 0,7801 & 1,5085 & 0,918 & 0,8925 & 0,9428 \\
\hline 20 & 0,949 & 1,206 & 0,8887 & 1,5224 & 0,964 & 0,9407 & 0,9869 \\
\hline 21 & 0,948 & 1,547 & 1,2331 & 1,8609 & 0,965 & 0,9411 & 0,9880 \\
\hline 22 & 0,933 & 2,095 & 1,7496 & 2,4400 & 0,946 & 0,9193 & 0,9720 \\
\hline 23 & 0,907 & 2,596 & 2,2010 & 2,9902 & 0,924 & 0,8937 & 0,9550 \\
\hline 24 & 0,892 & 2,801 & 2,3803 & 3,2224 & 0,920 & 0,8870 & 0,9534 \\
\hline média & 0,8668 & 1,5956 & 1,0853 & 2,1059 & 0,8742 & 0,8395 & 0,9089 \\
\hline
\end{tabular}

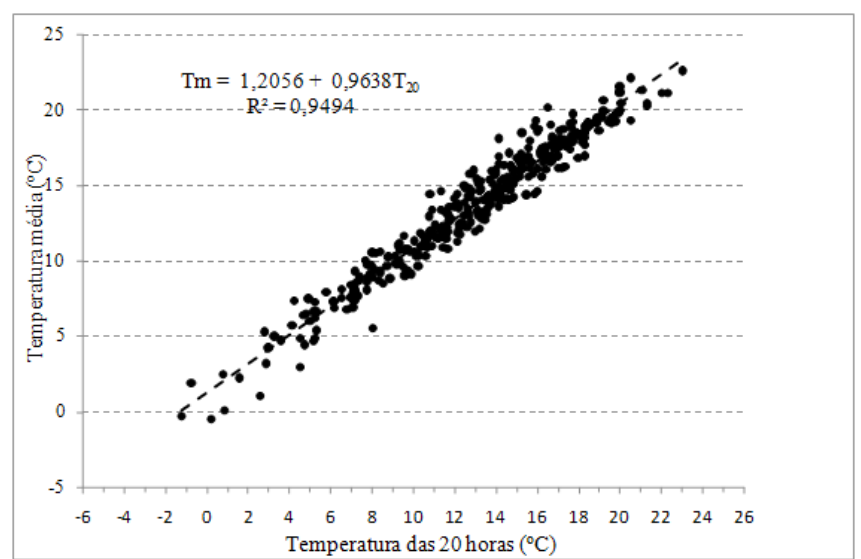

Figura 5 - Relação entre a temperatura média e a temperatura das 20 horas para São Joaquim-SC.

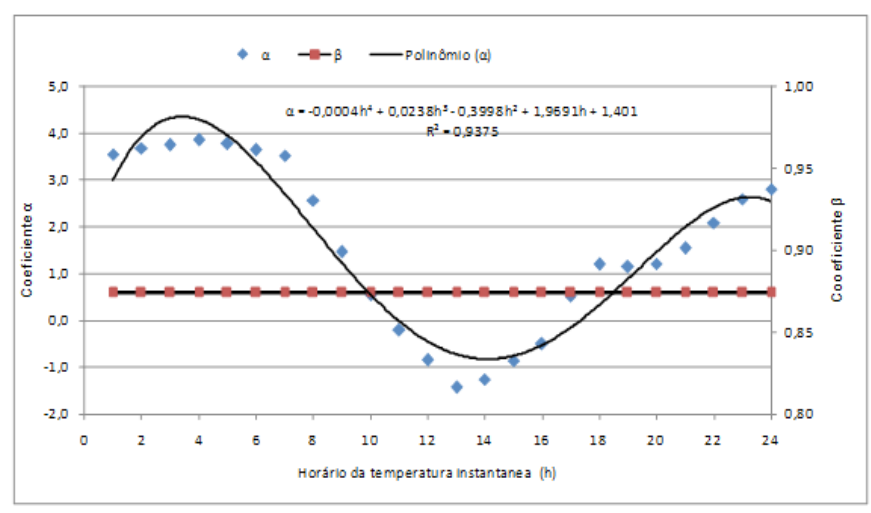

Figura 6 - Coeficientes $\alpha$ e $\beta$ da regressão entre a temperatura média diária e diferentes temperaturas de horários. 


\section{CONCLUSÕES}

Para se estimar a média diária das temperaturas diurnas (TD) pode-se utilizar as temperaturas instantâneas das estações meteorológicas convencionais ou automáticas, tendo as temperaturas das 11 horas o mais alto coeficiente de determinação.

Para se estimar a média diária das temperaturas noturnas (TN) pode-se utilizar as temperaturas instantâneas das estações meteorológicas convencionais ou automáticas, tendo as temperaturas das 24 horas o mais alto coeficiente de determinação.

Para se estimar a temperatura média diária (Tm) pode-se utilizar as temperaturas instantâneas das estações meteorológicas convencionais ou automáticas, sendo a melhor estimativa obtida com a temperatura das 20 horas.

Por meio da temperatura instantânea (Th), em qualquer horário (h), podem-se estimar as temperaturas noturnas (TN), diurnas (TD) e médias diárias (Tm) em São Joaquim com boa precisão.

\section{REFERÊNCIAS BIBLIOGRÁFICAS}

ARAUJO, M. I. M.; RODRIGUES, J. E. Estudo comparativo entre os dados coletados na estação climatológica principal de Brasília e uma estação automática. In: CONGRESSO BRASILEIRO DE METEOROLOGIA, 10, 1998, Brasília. Anais..., Brasília: Sociedade Brasileira de Meteorologia, 1998. (CD-ROM).

BRUNINI, O; SANTOS, J. M. dos; ALFONSI, R. R. Viabilidade técnica e performance de uma estação meteorológica automática. In: CONGRESSO BRASILEIRO DE AGROMEOROLOGIA, 10, 1997, Piracicaba, SP. Agrometeorologia, monitoramento ambiental e agricultura sustentável: Anais... Piracicaba: Sociedade Brasileira de Agrometeorologia, 1997. p.249-251.

CAMARGO, M. B. P.; BRUNINI, O.; MIRANDA, M. A. C. Temperatura-base para cálculo dos graus-dia para cultivares de soja em São Paulo. Pesquisa Agropecuária Brasileira, Brasília, v.22, n.2, p.115-121, 1987.

CONCEIÇÃO, M.A.F.; TONIETTO, J. Climatic potential for wine grape production in the tropical North Region of Minas Gerais State, Brazil. Revista Brasileira de Fruticultura, Jaboticabal, v.27, n.3, p.404-407, 2005.

CUNHA, A.R.; MARTINS, D. Estudo comparativo entre elementos meteorológicos obtidos em estações meteorológicas convencional e automática em Botucatu, SP, Brasil. Revista Brasileira de Agrometeorologia, Santa Maria, v. 12, n. 1, p. 103-111. 2004.

FERREIRA, E. A,;GALVANI, E.; ASSUNÇÃO, H. F. Avaliação comparativa entre os dados da estação convencional e automática da Fazenda Água Limpa/UnB - DF: In: CONGRESSO BRASILEIRO DE METEOROLOGIA, 12 ., 2002, Foz do Iguaçu. Anais..., Foz do Iguaçu: Sociedade Brasileira de Meteorologia, 2002. (CD-ROM).

FietZ, C. R.; LAZZAROTTO, C.; URCHei, M. A. Análise Comparativa preliminar de observações meteorológicas convencionais e automáticas em Dourados-MS. In: CONGRESSO BRASILEIRO DE AGROMETEOROLOGIA, 11, REUNIÃO LATINO AMERICANA DE AGROMETEOROLOGIA, II,1999, Florianópolis, SC. Anais. p.907-911.

FISCH, G.F; SANTOS, J. M. Comparações entre observações meteorológicas convencionais e automáticas na região do Vale do Paraíba, SP. In: CONGRESSO BRASILEIRO DE AGROMEOROLOGIA, 10., 1997, Piracicaba, SP. Agrometeorologia, monitoramento ambiental e agricultura sustentável: Anais. Piracicaba: Sociedade Brasileira de Agrometeorologia, 1997. p.246-248.

MURAKAMI, K.R.N.; CARVALHO, A.J.C. de; CEREJA, B.S.; BARROS, J.C.S.M.; MARINHO, C.S. Caracterização fenológica da Videira cv Itália (Vitis vinifera L.) sob deferentes épocas de Poda na Região Norte do Estado do Rio de Janeiro. Revista Brasileira de Fruticultura, Jaboticabal, v.24, n.3, p.615-617, 2002.

PEDRO JUNIOR, M. J.; SENTELHAS, P. C.; POMMER, C. V.; MARTINS, F. P. Determinação da temperatura base,graus dia e índices biometeorológicos para vieira Niágara Rosada. Revista Brasileira de Agrometeorologia, Santa Maria, v.2, p.51-56, 1994.

ROBERTO, S.R.; SATO, A. J.; BRENER, E. A.; JUBILEI, B.S.; SANTOS, C. E.; GENTA, W. Caracterização da fenologia e exigência térmica (graus-dias) para uva Cabernet sauvignon em zona tropical. Acta Scietiarum Agronomy, v.27, n.1, p.183-187, 2005.

SCARPARE, F. V.; OLIVEIRA, G. X. S.; SIMON, J.; PIEDADE, S. M. S.; ANGELOCCI, L. R. Comparação da uniformidade de dados meteorológicos de estação meteorológica convencional e automática - Piracicaba/SP. In: 14 CONGRESSO BRASILEIRO DE METEOROLOGIA, 2006, Florianópolis. Sociedade Brasileira da Meteorologia, 2006.

SENTELHAS, P.C.Análise comparativa de dados meteorológicos obtidos por estações convencional e automática. Revista Brasileira de Agrometeorologia, Santa Maria, v.5, n.2, p.215-221. 1997.

SENTELHS, P.C. Aspectos climáticos para viticultura tropical. Informe Agropecuário, v.19, n.194, p.9-14, 1998.

SOUZA, I. A; GALVANI, E.; ASSUNÇÃO, H.F. Avaliação de elementos meteorológicos monitorados por estações 
convencionais automática: In: CONGRESSO BRASILEIRO DE METEOROLOGIA, 12., 2002, Foz do Iguaçu. Anais..., Foz do Iguaçu: Sociedade Brasileira de Meteorologia, 2002. (CD-ROM).

TONIETTO, J. Les macroclimat viticoles mopndiaux et l'influencedu mésoclimat sur la typicité de la Syrah et du Muscat de Hambuourg dans desud de la France. Métodologie de caractérisation. 1999 233f. Tese (Doutorado) -École Nationale Supeérieure Agronomique de Montepllier, Montepellier.
TONIETTO, J.; CARBONNEAU, A. A multicriteria climatic classification system for grape-growing regions worldwide. Agricultural and Forest Meteorology, Amsterdam, v.124, p81-97, 2004. 